\title{
Promoting Positive Health Outcomes in an Urban Community-Based Physical Activity Intervention for Preschool Aged Children on the Autism Spectrum
}

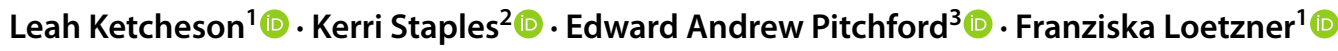

Accepted: 3 January 2021 / Published online: 18 January 2021

○ The Author(s), under exclusive licence to Springer Science+Business Media, LLC part of Springer Nature 2021

\begin{abstract}
While there is wide consensus regarding the importance of early intervention, health is rarely considered within priorities. Twenty-five children on the autism spectrum $\left(M_{\mathrm{age}}=4.67, \mathrm{SD}=0.82\right)$ participated in a 12-week physical activity intervention. Primary objective was to examine impact of a physical activity intervention on physical activity, fitness and motor competence. Secondary objective was to examine associations between motor behavior and ASD symptoms. Ball skills $(p<.001)$ and isometric push-up performance $(p=.02)$ improved. Autism symptoms were associated with motor skills $(r>-.49$, $p<.05$ ). Study outcomes provide new knowledge regarding design, delivery, and measures for early interventions targeting health disparities in young children on the autism spectrum.
\end{abstract}

Keywords Autism $\cdot$ Motor behavior $\cdot$ Health $\cdot$ Intervention

Children on the autism spectrum experience greater health disparities when compared to their neurotypical peers (Broder-Fingert et al. 2014; Curtin et al. 2005; Healy et al. 2019; Hill et al. 2015; Levy et al. 2019). In fact, a recently published report suggests that children on the autism

Supplementary Information The online version contains supplementary material available at https://doi.org/10.1007/s1080 3-021-04871-7.

Leah Ketcheson

Leah.ketcheson@wayne.edu

Kerri Staples

skerri@umich.edu

Edward Andrew Pitchford

Eapitch@iastate.edu

Franziska Loetzner

Loetzner@wayne.edu

1 Department of Kinesiology, Health and Sport Studies, Wayne State University, 5101 John C Lodge Fwy, 257 Matthaei, Detroit, MI 48202, USA

2 School of Kinesiology, Center On Physical Activity \& Health in Pediatric Disabilities, University of Michigan, 1402 Washington Hts, Ann Arbor, MI 48109, USA

3 Department of Kinesiology, Iowa State University, 534 Wallace Road, 103n Forker, Ames, IA 50011, USA spectrum experience disproportionate rates of overweight and obesity beginning as early as 2 years of age (Levy et al. 2019). This is concerning given the growing prevalence of Autism Spectrum Disorder (ASD). It is currently estimated that 1 in every 54 children are diagnosed with the disorder (Maenner et al. 2020). One of the most broadly disseminated messages regarding treatment practices suggests that services begin before 3 years of age (National Autism Center, 2009a, b). While there is wide consensus regarding the importance of early intervention, health is rarely considered within the targeted priorities. Unfortunately, many children on the autism spectrum have qualitatively and quantitatively different experiences (Bhat et al. 2011) which can oftentimes lead to impairments across several domains of development including their motor behavior. Specifically, children on the autism spectrum experience significant impairments across multiple health domains including the performance of fundamental motor skills (FMS; Jasmin et al. 2009; Lloyd et al. 2013; Provost et al. 2007; Staples and Reid 2010), physical activity (PA; Gehricke et al. 2020; Gillette et al., 2015; MacDonald et al. 2011), and health related physical fitness (HRPF; Staples et al. 2020). The presence of impairments in any one of these domains can persist, oftentimes creating negative health consequences throughout development including obesity and physical inactivity (Robinson et al. 2015; Stodden et al. 2008). A greater understanding of 
health disparities will elucidate the need for targeted interventions that promote positive trajectories of health.

For the purpose of this study, motor behavior including; FMS, PA and HRPF will be reviewed and examined in relation to the overall health and well-being in children on the autism spectrum. Therefore, an important distinction in motor behavior terminology will first be described. FMS includes both ball (e.g. strike, kick, dribble) and locomotor (e.g. skip, hop, run) skills (Ulrich 2018). A requisite proficiency in FMS affords children the opportunity to participate in PA (Logan et al. 2015). Next, the term PA is typically defined as participation in one of five levels (e.g. sedentary, light, moderate, moderate to vigorous [MVPA] and vigorous). When in relation to health outcomes, the most commonly cited reference is MVPA. National recommendations suggest school age youth (aged 6-17 years) should participate in a minimum of $60 \mathrm{~min}$ of MVPA each day, as this is related to positive health outcomes [Physical Activity Guidelines (PAG) 2018]. Moreover, preschool aged children should be physically active throughout the day as a way to promote healthy growth and development (PAG 2018). Finally, HRPF encompasses cardiorespiratory fitness, musculoskeletal fitness, flexibility, and balance (PAG 2018). It is an integral component of health to consider given it is related to an individual to perform daily tasks and enjoy leisure time pursuits (PAG 2018). Finally, motor behavior will be used as an overarching term that includes each component of health within reference to early screening or intervention targets for children on the autism spectrum.

The rates of overweight and obesity in school aged children have steadily increased over the past several decades (Ogden et al. 2016). However, even more disparaging is the prevalence of unhealthy weight classifications among children on the autism spectrum. When examining health indices, one of the most commonly reported metrics is weight classification. Obesity is classified by body mass index (BMI) and is calculated as weight in kilograms divided by the square of height in meters $\left(\mathrm{kg} / \mathrm{m}^{2}\right)$. Childhood weight classifications are determined with BMI-for-age percentiles, which examine a child's BMI score relative to children of the same age and sex using the CDC growth charts (2000). Children who are overweight have a BMI-for age that is greater than or equal to the 85 th percentile, and children who are obese have a BMI-for-age at or above the 95th percentile. Current research suggests children on the autism spectrum are more likely to be classified as overweight or obese compared to their neurotypical peers (Healy et al. 2019 (10-17y), Hill et al. 2015 (2-17y); Broder-Fingert et al. 2014 (2-20y); Zuckerman et al. 2014). More specifically, 2-5-year-old children on the autism spectrum are 1.57 times more likely to be overweight or obese than neurotypical children of the same age (Levy et al. 2019). Further, cross-sectional research examining children on the autism spectrum between the ages of 2-5 and 6-11 years suggests an increasing prevalence of overweight and obesity with increasing age (Curtin et al. 2005; Hill et al. 2015). Moreover, the prevalence of an unhealthy weight status persists throughout adolescence and into early adulthood, with the prevalence of being overweight or obese further increasing between the ages of 6-11, 12-15, and 16-20 years of age (Broder-Fingert et al. 2014). Collectively, the disparities in weight classifications emerge very early on in development, persist throughout childhood (Broder-Fingert et al. 2014) and disproportionately impact children on the autism spectrum (Broder-Fingert et al. 2014; Healy et al. 2019; Hill et al. 2015; Levy et al. 2019). However, understanding factors contributing to unhealthy weight classification elucidate the need for targeted interventions that move beyond core deficits. One plausible explanation for health disparities among children on the autism spectrum are the clear absence of interventions addressing health outcomes in this population.

Children on the autism spectrum exhibit pervasive delays in motor behavior beginning early on in development (Staples et al. 2012). In fact, there is retrospective and prospective research examining differences in the rate and quality of motor milestone acquisition within the first year of life (Teitelbaum et al. 1998; Flanagan et al. 2012). The gross motor delays in toddlers on the autism spectrum emerge as young as 1 year of age, and when examined longitudinally delays continue to increase between 2 and 3 years of age (Lloyd et al. 2013). This slowed rate of development is interesting to consider given the pervasiveness of delays which persists throughout early childhood. In one such study, motor delay in young children less than 4 years of age including a group of children on the autism spectrum, was examined with a comprehensive measure of early motor development (Provost et al. 2007). Young children had significantly lower gross, fine, and total motor quotients compared to peers of similar age (Provost et al. 2007). Most concerning was that $68 \%$ of children on the autism spectrum qualified for early intervention by 3 years of age based on their motor delay alone (Provost et al. 2007). Using the same measure, the results of a separate study demonstrate that 3 to 4 year-old children on the autism spectrum experience significant impairments in both gross and fine motor quotient scores (Jasmin et al. 2009). Where 63\% of children experienced a significant gross motor delay when compared to what would be expected for their chronological age (Jasmin et al. 2009). When taken together, these findings suggest impairments in motor behavior persist beyond infancy and are evident throughout preschool age (Provost et al. 2007; Jasmin et al. 2009). By school age children on the autism spectrum perform FMS with similar levels of competence to children half their chronological age (Staples and Reid 2010). Collectively, this is concerning given ball and locomotor skills are considered the building blocks of future motor behavior 
participation, including participation in PA (SHAPE America 2014). While the translation of motor milestones to FMS delay needs to be explored longitudinally, there is ample research evidence that children on the autism spectrum demonstrate pervasive delays in the motor domain.

Because motor skills and PA contribute to positive trajectories of health (Robinson et al. 2015; Stodden et al. 2008), they should be an important consideration with the early intervention for children on the autism spectrum. National recommendations from the Society for Health and Physical Education (SHAPE America 2014) suggest preschool aged children should be afforded ample opportunity to develop proficiency in fundamental movement skills as they serve as the basis for future motor skillfulness and participation in physical activity. As a result, much of the published research promoting physical activity early on in development focuses on the acquisition of fundamental motor skills including locomotor skills (e.g.: run, jump, skip) and ball skills (e.g.: kick, strike, throw) (Logan et al. 2012). To date, there is limited consensus regarding participation in health enhancing PA in children on the autism spectrum. Ketcheson et al. (2018) reported that even after controlling for gross motor skills, preschool aged children on the autism spectrum (aged 4-6) participated in significantly more MVPA than their same age neurotypical peers. While these findings are interesting, authors did not explore how the study sample accumulated the PA. In other words, the role of stereotypical behaviors on the accumulation of PA may have inflated the contribution of health enhancing participation in PA. In a separate study, Thomas et al. 2019 examined participation in MVPA in a group of children with and without autism spectrum disorder aged 4-7, and discovered no between groups differences with regards to participation in MVPA. However, authors suggest further exploration into the ecological correlates (e.g. familial and environmental) and participation in PA (Thomas et al. 2019). The measurement of PA in preschool aged children on the autism spectrum remains an area of research that requires further clarity regarding the interpretation of objectively measured PA. To date, measurement of PA in school aged youth is limited to cross-sectional research and parent report. Declining levels of PA from childhood to adolescence has been reported (MacDonald et al. 2011). Moreover, when compared to neurotypical peers, children on the autism spectrum, aged 6-11, participate in significantly less daily PA (Gehricke et al. 2020). Regardless, participation in a requisite amount of MVPA is a known contributor to the promotion of healthy trajectories in children, and the promotion of PA participation early on in development may help to curb the onset of health disparities in young children on the autism spectrum.

Next, there remains a scarcity of research examining the levels of HRPF in young children on the autism spectrum. Although the majority of 7-12 year old children with intellectual and developmental disabilities scored below the age-specific standards for each fitness task (Winnick and Short 2014), they did demonstrate significantly improved cardiovascular and musculoskeletal fitness following participation in a 10-week PA program (Collins and Staples 2017). The results of this study demonstrate the feasibility of measuring fitness with children on the autism spectrum, but the lack of a comparison group limits the application and generalization of these results. Compared to neurotypical children of the same age, 6-9 year old children with ASD had significantly lower levels of muscular strength and endurance and cardiovascular fitness (Staples et al. 2020). Collectively, research examining the aforementioned components of health are important to consider given relationships among FMS, PA and HRPF interact and strengthen over time, and together are key factors that can influence trajectories of health (Stodden et al. 2008; King-Dowling et al. 2020).

The benefits of timely interventions beginning early on in development are key to achieving optimal outcomes. Despite motor behavior being named as 1 of 8 domains to be targeted in the early intervention for children on the autism spectrum (National Autism Center 2009b), there remains a sparsity of published data addressing motor and health behaviors prior to school age. Moreover, research examining the effectiveness of interventions has been limited mostly with regards to singular outcome variables with little consideration of cascading effects between developmental domains (Srinivasan et al. 2014). In a school-based intervention, children on the autism spectrum aged 5-12 years old participated in 3, 40-min sessions per week for 12 weeks of Sports, Play, and Active Recreation for Kids (SPARK) as part of their physical education curriculum. Following $24 \mathrm{~h}$ of instruction children on the autism spectrum had significantly improved scores on both static and dynamic balance (Najafabadi et al. 2018). In a separate study, a 24- week exercise program that included $16 \mathrm{~h}$ of total instruction was delivered to children on the autism spectrum aged 6-12 years (Toscano et al. 2018). Following participation, children demonstrated improved metabolic health as measured by levels of high- and low-density lipoprotein and total cholesterol (Toscano et al. 2018). While these findings are promising with respect to long term health or risk for chronic disease, outcomes were limited to physiological changes with very little reported on improvements to components of health integral to participation in PA.

Beyond improvements to health that participation in PA are afforded, there is a small but emerging area of research examining relationships among physical activity and socialization. Current findings suggest relationships among the gross motor performance and social skills are present early on in development and persist throughout childhood. Greater social communication delays were discovered in toddlers on the autism spectrum with poorer motor proficiency 
(MacDonald et al. 2013). Similarly, in a separate study that examined relationships among children on the autism spectrum 18 months through 6 years of age, children who demonstrated the most significant gross motor impairments also exhibited the poorest scores in socialization (Pusponegoro et al. 2016). Moreover, correlations among objectively measured motor skills and parental report of social responsiveness suggest children on the autism spectrum aged 4-12, with lower subscale motor scores were related to impairments in social skills (Gladfelter et al. 2020). As children acquire a threshold of proficiency in fundamental motor skills it is likely they will be afforded additional opportunities for structured (e.g. adapted physical education) and unstructured play (e.g. recess) play. Over developmental time, these opportunities will create positive spirals of engagement, where children with greater motor proficiency will continue to participate in recreational pursuits of physical activity including community-based programming. Next, positive social outcomes have also been reported following the delivery of PA interventions to young children on the autism spectrum. Among improvements cited are reduced disruptive behaviors (Pan 2010), improved peer interactions (Ketcheson et al. 2017) and improvements to individual participant social skills (Bremer et al. 2015). Finally, in a meta-analysis examining the effects of PA interventions on young individuals on the autism spectrum (aged 2-22), results support the effectiveness of intervention for improvements in FMS (e.g. locomotor skills), physical fitness (e.g. muscular strength and endurance) and social functioning (Healy et al. 2018). The majority of intervention studies addressing the motor behavior in individuals on the autism spectrum include samples with large age ranges, making individualized instruction difficult. Moreover, many interventions begin during the school years, missing opportunities to intervene during early developmental periods when children may be most sensitive to change. Collectively, these studies support the promising impact of interventions that target component(s) of health. However, there remains a significant void of interventions that comprehensively promote and address positive trajectories of health beginning early on in development.

While mounting evidence suggests children on the autism spectrum could benefit from interventions targeting health outcomes, another often overlooked opportunity to promote developmental trajectories is the relationship between PA and social competency. Given participation in PA has been identified as a potential gateway to improve social skills (Bremer et al. 2015; Ketcheson et al. 2017; Pan 2010), it seems possible that children on the autism spectrum (who experience pervasive social-communication deficits) may benefit across multiple domains when participating in interventions that promote PA. Few studies to date have delivered PA interventions and reported improvements to social competencies (Bremer et al. 2015; Ketcheson et al. 2017; Pan 2010). Implementing PA interventions to improve social communication outcomes in preschool children on the autism spectrum remains an area of future research that warrants greater attention, especially in the methodology and delivery of comprehensive early interventions.

Children on the autism spectrum experience health disparities at a disproportionately higher rate when compared to their neurotypical peers. When compared to same age neurotypical peers, children on the autism spectrum experience lower proficiency in FMS (Jasmin et al. 2009; Lloyd et al. 2013; Provost et al. 2007; Staples and Reid 2010), low levels of physical activity (Gehricke et al. 2020; Gillette et al. 2015; MacDonald et al. 2011) and lower levels of HRPF (Staples et al. 2020). A key contributor to unhealthy weight classifications are the well documented lack of the opportunities for children on the autism spectrum participate in community-based PA programs and health promotion interventions. Based on the currently available published research, when interventions are delivered to children on the autism spectrum, they generally lack the combination of strong methodology and comprehensive measures of health. To address this gap in the literature, a physical activity intervention was designed and delivered to children on the autism spectrum where outcomes across multiple components of health were evaluated. Specifically, the primary objective of this study was to examine the impact of a 12-week community based physical activity on multiple components of health (e.g. weight classification, FMS, PA and physical fitness). Our secondary objective was to examine social determinants of motor behavior (including motor skills, physical fitness, and PA) and social communication scores at baseline, and to examine if improvements in motor behavior were associated with social communication scores. Collectively, the knowledge gained from this study will influence the design and delivery of PA programming as a key component of early intervention efforts to improve health outcomes for young children on the autism spectrum.

\section{Methods}

\section{Participants}

Dissemination of study information occurred through social service networks, including parent advocacy groups, and early intervention service providers. To be included in this study, participants had a confirmed diagnosis of ASD (by school or medical provider), resided within 15 miles of the study site, and were between a narrow age range 3 and 5 years of age. A group of 32 children with ASD was recruited for the study, but 25 participants completed the 12 week intervention and both pre and post measurements. 
Attrition was due to inability to complete baseline measurements $(n=5)$, withdrawal due to parental decision $(n=2)$, and change in diagnosis affecting inclusion/exclusion status $(n=1)$. As can be observed in sample characteristics (see Table 2), the participants consisted of a diverse group of children, including 50\% identifying as African American.

\section{Descriptive Measures and Diagnostic Instruments}

In order to establish a baseline understanding of intelligence quotient (IQ) and to afford direct comparisons to other research examining the effectiveness of communitybased early intervention (Nahmias et al. 2019), all research participants were administered the Mullen Scales of Early Learning (MSEL; Mullen 1995). Each of the four cognitive scales: receptive and expressive language, non-verbal problem solving, and fine motor skills were administered. While the MSEL also includes a gross motor component, we did not administer given a more comprehensive evaluation of motor behavior would subsequently be administered. When possible, age equivalent scores on the expressive language subscale were generated as a method to assist researchers with the selection of the appropriate Autism Diagnostic Observation Schedule -2 (ADOS-2; Lord et al. 2012). The ADOS-2 were administered by one of two faculty members who were research reliable in the administration and scoring of the assessment. The ADOS- 2 is a standardized observational assessment that includes five modules; the selection of the module is based on the child's expressive language level (Lord et al. 2012). Children in the current study were administered a module 1 for children with little or no phrase speech or Module 2, for children who use phrase speech but are not yet fluent. Standardized domain scores for the ADOS were computed (as symptoms of severity) in two domains, social affect (SA) and restricted repetitive behaviors (RRB). As an indicator of ASD severity for children on comparison scores were generated based on a sum and subsequent conversion of scores from the $\mathrm{SA}$ and $\mathrm{RRB}$, and were used as an indicator of ASD severity for children (Gotham et al. 2009). A comparison score between 1 and 2 indicates minimal to no evidence, 3-4 minimal evidence, 5-7 moderate evidence and $8-10$ high evidence.

\section{Outcome Variables}

The Test of Gross Motor Development-3 (TGMD-3; Ulrich 2018) is a norm referenced evaluation of gross motor skills and is one of the most widely employed assessments of FMS. In the availability of motor behavior research for children on the autism spectrum, it remains one of the most utilized assessments (Bremer et al. 2015, 2016; Ketcheson et al. 2017, 2018, 2020; Staples et al. 2012). It is considered a valid and reliable assessment of gross motor skills for children ages 3-10. Recent research has also established the instructional sensitivity of the TGMD-3, where raw scores are sufficiently sensitive to detect changes in performance following 10 weeks of physical education for young children (Staples et al. 2020). Moreover, it is appropriate to use in research for children on the autism spectrum given its inclusion of clinical populations in its standardized normative sample with established clinical validity for children with ASD (Pitchford and Webster 2020; Ulrich 2018). Next, the skills in the TGMD-3 are age and developmentally appropriate for children in this study, in other words, children typically have fun engaging in the type skills represented in the TGMD-3. Briefly, the TGMD-3 is comprised of two subscales, locomotor (e.g. run, jump, skip) and ball (e.g. kick, strike, throw) skills. The TGMD-3 manual includes procedures for a standardized administration of the evaluation which includes verbal details along with a physical demonstration. All participants were provided a demonstration followed by a practice trial and two scored trials. Additionally, it should be noted that in the testing manual flexibility in administration procedures to elicit a child's most representative behavior is encouraged. Given the behavioral challenges that can be presented in testing situations, the TGMD-3 affords the greatest amount of information in relation to time to administer. All TGMD-3's were recorded and scored by two research assistants with ample experience in conducting standardized assessments in young children on the autism spectrum. To calculate inter-reliability for the scoring of the TGMD-3, 10 of the 25 (40\%) assessments were scored by two independent raters at both time points. Intraclass correlation coefficients (ICC) were calculated using a two-way random effects model (ICC 2,1 ) that examined the absolute agreement between raters. The average ICC for each subtest score across both time points for the locomotor and ball skills subtests were 0.998 (0.981-0.999) and 0.994 (0.978-0.999), respectively. Both TGMD-3 raw scores and standardized scores were used in analyses. Raw scores, as utilized in the primary analyses, reflect the absolute change in fundamental motor skill proficiency and are more sensitive to detecting change than standardized scores. The gross motor index and standardized subscale scores reflect a norm-referenced evaluation of performance compared to children in the United States based on age and sex.

The HRPF of participants was measured with 4 tasks from the Brockport Physical Fitness Test (Winnick and Short 2014). The selected tasks are considered appropriate measures of HRPF for children on the autism spectrum given and have been used previously in research with children with developmental disabilities (Collins and Staples 2017; Staples et al. 2020). The study by Collins and Staples (2017) also demonstrate these measures are sufficiently sensitive to detect changes in HRFP capacities following participation in a 10- week PA program. 
Similar to the TGMD-3 administration protocol, all participants were provided a demonstration with verbal instructions, followed by a practice trial. Measures of muscular strength and endurance included the isometric push up (upper body), the modified curl up (abdominal), and the standing long jump (lower body). For the standing long jump, each child completed 2 trials and the farthest jump was recorded. The $20-\mathrm{m}$ progressive aerobic cardiovascular endurance run (PACER) measured the cardiovascular endurance of each research participant. A research assistant ran alongside each participant for the PACER so each child could see and hear the pace at all times.

The PA of children was evaluated using Actigraph GT3X + (Pensacola, FL), which are small accelerometers that measure levels of PA in three planes of movement. They are considered the gold standard for evaluation of PA given their ease of use and objective methodology. Parent(s) were instructed to administer the accelerometers around the participants waist above the right iliac crest, during all waking hours (except during bath or shower) for 7 days including 2 weekend days. Following wear time recommendations for PA measurement (Matthews et al. 2012; Rich et al. 2013), children with $\geq 2$ days of $\geq 10 \mathrm{~h}$ of wear time were included in the analysis. The majority (92\%) of children had either $\geq 3$ valid days of $\geq 10 \mathrm{~h}$ of wear time or $\geq 4$ days of $\geq 8 \mathrm{~h}$ of wear time. A valid day for this age group has previously been defined as $\geq 5 \mathrm{~h}$ of wear time and $\geq 4$ valid days (Pfeiffer et al. 2009). Participants wore the accelerometers 1-week pre intervention and 1-week post intervention. This time represented a warm and cold period respectively in the region where the study was conducted. Accelerometer data was collected and was downloaded with Actilife 6 software using $5 \mathrm{~s}$ epochs. Validated and published cutpoints by Butte (2014) were used to quantify participant movement in 5 intensities of activity; sedentary $(<240 \mathrm{CPM})$, light PA, (240-2119 CPM), moderate PA (2120-4449 CPM), and vigorous PA ( $>4450 \mathrm{CPM}$ ). MVPA is the sum of activity counts accumulated in the moderate and vigorous intensity categories to provide an indication of PA levels relative to the US PA guidelines (PAG 2018). Of the 25 children in the sample, 18 completed both pre and post PA measurements.

Body composition was evaluated by administering height and weight as well as waist to hip ratio. BMI $\mathrm{z}$-scores were calculated based on participant height and weight (BMI: $\mathrm{kg} / \mathrm{m} 2$ ) and were used in our analysis as the primary variable of body composition (Kuczmarski et al. 2000). Z scores are measures of relative weight adjusted for child age and sex allowing for evaluation of growth status independent of other factors.

\section{Intervention}

The intervention was originally designed as a two-year longitudinal intervention, where participants would have received dosage in six 12-week blocks. Pre and post measures were planned throughout the longitudinal intervention and would have resulted in a trajectory of change with 6 timepoints of measures. However, due to social distancing circumstances that arose with COVID-19, the methodology for the intervention was altered to a pre and post design consisting of 12, 1-h sessions per week. The intervention followed a consistent routine each day (see Table 1). All intervention sessions were conducted in a gymnasium in a large and public urban university located in Southeast Michigan. The primary focus of the intervention was on the acquisition and reinforcement of the 13 fundamental motor skills that are included in the TGMD-3. Given best practices in instructional methods for physical education (Michigan Fitness Foundation: Michigan's Exemplary Physical Education Curriculum 2002), two skills (1 locomotor and 1 ball) were selected each session as the primary focus.

The intervention included two different types of instructors; coaches who worked in 1:1 dyads with research participants, and lead teachers. All coaches were pre-service health and physical education teachers enrolled in an adapted physical education college course with an embedded practicum component. Their role primarily consisted of direct individualized instruction on motor behavior skills during individual, small and large group activity. For consistency, coaches remained paired with the same participant for each of the 12 sessions. Next, two lead teachers with Board Certified Behavioral Analysts (BCBA's) credentials were responsible for the management, timing and delivery of group direction. Moreover, once activities began, the lead teachers circulated to coach and participant dyads in order to provide feedback that promoted participant time on task. The principal investigator (PI) is a certified adapted physical education teacher with over 15 years of experience conducting community based adapted PA programming.

Every session began with a social story warm up where opportunities to practice each of TGMD-3 ball and locomotor skills was presented. Next, the lead teachers provided an overview of the session, where action pictures of subsequent activities were presented in chronological order. Following a review of session activities, the lead teachers assigned task cards to all dyads. Task cards were designed with a series of progressively difficult activities that participants could complete with their assigned coach. The purpose of the cards was to progress on previously introduced TGMD-3 activities. For example, if the stationary kick was introduced in the session prior, the task card would begin with a review of the skill previously acquired (e.g. swing leg to contact ball with force from foot), followed by an activity requiring greater 
Table 1 Intervention session outline

\begin{tabular}{|c|c|c|c|}
\hline Hour & Overview & TGMD-3 skill (example) & CPRT strategy \\
\hline $0-5 \mathrm{~min}$ & Warm up & $\begin{array}{l}\text { Lead teachers read and acted out a social story } \\
\text { with embedded opportunities to practice each } \\
\text { of the TGMD-3 skills }\end{array}$ & Student attention, multiple cues \\
\hline $5-15 \min$ & Task card & $\begin{array}{l}\text { Coach and child dyads completed task cards that } \\
\text { consisted of a review of previously acquired } \\
\text { skills, along with progressively difficult task } \\
\text { requirements }\end{array}$ & $\begin{array}{l}\text { Easy and difficult tasks, direct reinforcement, } \\
\text { shared control }\end{array}$ \\
\hline $15-18 \min$ & Introduce 2 new skills & $\begin{array}{l}\text { Lead teachers demonstrated } 2 \text { new skills along } \\
\text { with key words, dyads were encouraged to find } \\
\text { space in the gym to practice motor movements } \\
\text { and key words }\end{array}$ & $\begin{array}{l}\text { Clear and appropriate instruction, reinforcement } \\
\text { of attempts }\end{array}$ \\
\hline $18-20 \mathrm{~min}$ & Introduce small group activity & $\begin{array}{l}\text { Lead teachers divided small groups according to } \\
\text { skill level, and assigned to designated area of } \\
\text { gymnasium, for example wall A, B, C or D }\end{array}$ & Clear and appropriate instruction \\
\hline $20-35 \mathrm{~min}$ & Small group activity & $\begin{array}{l}\text { Coaches worked with children to complete } \\
\text { the small group activity, coaches provided } \\
\text { feedback regarding turn taking or behavioral } \\
\text { re-direction during goal-oriented task, the } \\
\text { activity included an opportunity to practice } \\
\text { both TGMD-3 skills }\end{array}$ & $\begin{array}{l}\text { Multiple cues, direct reinforcement and contin- } \\
\text { gent consequence }\end{array}$ \\
\hline $35-38 \mathrm{~min}$ & Water break & $\begin{array}{l}\text { Coaches provided an optional opportunity for } \\
\text { children to walk towards break station for a } \\
\text { drink of water }\end{array}$ & Shared control \\
\hline $38-40 \mathrm{~min}$ & Introduce large group activity & $\begin{array}{l}\text { Lead teachers divided large group into two, and } \\
\text { assigned to designated area of gymnasium, for } \\
\text { example, side one or two }\end{array}$ & Clear and appropriate instruction \\
\hline $40-55 \mathrm{~min}$ & Large group activity & $\begin{array}{l}\text { Lead teachers provided two to five-minute } \\
\text { opportunities for large groups to complete the } \\
\text { timed activity, the activity included an oppor- } \\
\text { tunity to practice both TGMD- } 3 \text { skills }\end{array}$ & $\begin{array}{l}\text { Multiple cues, direct reinforcement, } \\
\text { contingent consequence }\end{array}$ \\
\hline $55-57 \mathrm{~min}$ & Review & $\begin{array}{l}\text { Lead teachers asked several facilitating ques- } \\
\text { tions related to TGMD-3 skills }\end{array}$ & Student attention, reinforcement of attempts \\
\hline $57-60 \mathrm{~min}$ & Cool down & $\begin{array}{l}\text { Lead teachers provided instruction on yoga } \\
\text { poses for dyads to complete together within a } \\
\text { large group setting }\end{array}$ & Student attention \\
\hline
\end{tabular}

TGMD-3 Test of Gross Motor Development, 3rd edition (Ulrich 2018); CPRT Classroom Pivotal Response Teaching

skill proficiency (e.g. rapid approach to the ball prior to contact). Following the completion of task cards, the lead teachers introduced two new TGMD-3 skills to the group. The lead teachers then provided several minutes for the dyads to practice the TGMD-3 skills, including the reinforcement of key words (e.g. run, leap, kick) with corresponding motor movement (e.g. child kicks ball).

Next, the lead teachers divided dyads into several learning groups corresponding to their proficiency in the given skills. The groups usually consisted of participants who were either acquiring, practicing or refining skills, in this way, the small groups could complete the task requirements at a pace comfortable to their group. The small group activity was a goal-oriented task that encouraged participation from all group participants to complete. For example, the purple team was responsible for running to the purple cone and throwing the tennis balls into the purple bucket. Depending on overall timing, several opportunities to complete the goal-oriented task were afforded. Next, instructions were delivered for the large group activity which consisted of a time-oriented task. For example, all participants were instructed to run around the perimeter of the gym and complete as many target throws as they could within a designated time frame (e.g. $2 \mathrm{~min}$ ). Multiple opportunities to engage in the activity were afforded, where instructors increased the length of time participants engaged as a large group to complete the activity. Once the large group activity concluded, lead teachers delivered a short lesson review where facilitating questions regarding TGMD-3 skill criterion were posed to the group. For example, participants were asked to show or tell the lead teachers which foot should step forward when throwing a ball, or how to move the arms in opposition during the run. Finally, a social story cool down where 
opportunities to practice yoga poses requiring muscular strength and endurance was delivered.

\section{Behavioral Framework}

All instructors participated in a 3-h training on the behavioral framework called Classroom Pivotal Response Teaching (CPRT; Suhrheinrich et al. 2011). This behavioral framework has been used successfully in previous adapted physical activity intervention research (Ketcheson et al. 2017). The CPRT framework includes 5 antecedent (student attention, clear and appropriate instruction, easy and difficult tasks, shared control, and multiple cues), and consequence (direct reinforcement, contingent consequent and reinforcement of attempts) strategies. Instructor training consisted of a training on each of the CPRT strategies where the opportunity to practice and reinforce prompts within different instructional situations was operationalized. There were a total of 25 coaches who worked in 1:1 dyad with the research participants and were evaluated for fidelity on the CPRT strategies. The items on the fidelity assessment included the 5 antecedent and 3 consequence CPRT strategies. The PI evaluated the presence of a strategy with a ' 1 ' and the absence of a strategy with a ' 0 '. The PI tracked the frequency of fidelity checks to ensure all instructors received an equal opportunity to be evaluated for fidelity at the beginning (weeks 1- 4), middle (weeks 5-8) and end (weeks 9-12) of the duration of the intervention. The PI evaluated the performance of coaches in real time during the intervention sessions. Fidelity outcomes were discussed as a group at the conclusion of each session, all components of the CPRT were reviewed with the PI, and discussed relative to fidelity. In this way all coaches could continue to ensure participants received quality instruction.

\section{Procedures}

All study procedures were approved by the Institutional Review Board at a large urban university in Southeast Michigan. Pre-measures were evaluated over 2 separate meetings prior to study commencement. In the first meeting, approximately 1-month pre intervention, parents consented their child's participation in the study, and completed a questionnaire regarding their child's extracurricular and physical activity behavior (including community services, occupational and physical therapy). Following completion of the paperwork, all participants were administered the MSEL and the ADOS-2. Approximate language levels from the MSEL was used to select the appropriate ADOS-2 module.

In the second meeting, all components of health were administered to study participants one week prior to intervention commencement. Upon arrival research participants were paired with their instructional coach and were shown a picture schedule outlining each of the stations to be completed. The stations were set up around the perimeter of a large gymnasium, instructors were encouraged to allow the participant to select the order of activities. Each station was equipped with stickers and food reinforcers that were awarded (if needed) at the completion of each activity. Stations included height, weight and waist circumference, 20-m PACER, muscular strength and endurance, and ball and locomotor skills. To ensure minimal distractions within the gymnasium, participants were scheduled to arrive in a pre-arranged block of time to reduce the number of individuals in the gym at one time. Once all measures were completed, parents were provided the physical activity monitor along with instructions for adherence to wear time protocols. These procedures were operationalized again 1 week following the completion of the intervention.

\section{Statistical Procedures}

Descriptive statistics including mean and standard deviation or frequency and proportion were calculated from pre- and post-intervention measurements. Differences in outcome variables attributable to the intervention were analyzed using paired t-tests with Cohen's $d_{z}$ effect sizes $\left(\mu_{z} / \sigma_{z}\right)$. Analyses were conducted for changes in TGMD-3 raw scores, healthrelated physical fitness, and physical activity levels. Actigraphy data was missing from 7 of the 25 participants that completed the intervention. Paired t-tests for TGMD-3 and HRPF data were conducted with all 25 participants while PA change was conducted with 18 participants. Differences between the samples with and without PA data are presented in Supplementary Table S1. To determine the interrelatedness of these intervention components, Pearson correlation was used to examine bivariate correlation between variables at both baseline and with change scores $(\Delta)$ from pre to post intervention. As an exploratory analysis, Pearson correlation was used to examine bivariate associations between ASD symptoms, as measured by the ADOS-2, and the primary intervention outcomes at both baseline and with change scores $(\Delta)$. For correlational analyses, the standardized Gross Motor Index from the TGMD-3 was also incorporated to better account for the influence of age and gender. Statistical analyses were conducted in SPSS (v.26) with a priori alpha of 0.05 . Effect sizes $\left(d_{\mathrm{z}}\right)$ were calculated in $\mathrm{G}^{*}$ Power (v.3.1).

\section{Results}

Twenty-five children with ASD completed the intervention and both pre and post measurements. Participant attendance across the 12 week intervention ranged from 9 to 12 weekly sessions $(M=10.88 \pm 1.05 ; 90.67 \%)$. Descriptive statistics of 
the sample are reported in Table 2 . The sample was predominately male (72\%) and African American (52\%).

The intervention was effective in improving fundamental motor skills and heath related physical fitness in children with ASD (see Table 3). TGMD-3 ball skills scores $\left(M_{\text {change }}=5.28 \pm 5.62, p<0.001\right)$ had significant increases from pre to post test. However, gains in locomotor skills

Table 2 Demographic characteristics

\begin{tabular}{lll}
\hline & $N(\%)$ & $M(S D)$ \\
\hline$n$ & $25(100 \%)$ & \\
Age & & $4.67(0.82)$ \\
Male & & \\
Female & $18(72 \%)$ & \\
Race/Ethnicity & $7(28 \%)$ & \\
African American & & \\
Caucasian & $13(52 \%)$ & \\
Hispanic/Latino & $5(20 \%)$ & \\
Other & $1(4 \%)$ & \\
ADOS-2 & $6(24 \%)$ &
\end{tabular}

ADOS-2

Social Affect

$12.88(3.33)$

Restrictive \& repetitive behaviors

$3.84(1.80)$

Comparison score

$7.40(2.74)$

MSEL

Receptive language

$30.36(9.76)$

Expressive language

$29.36(9.36)$

Non-verbal problem serving

$34.64(9.52)$

Fine motor

$33.84(8.22)$

All demographic characteristics are presented as frequency (proportion) for categorical data and mean (standard deviation) for continuous data. ADOS-2: Autism Diagnostic Observation Schedule, 2nd edition (Lord et al., 2012); MSEL: Mullen Scales of Early Learning (Mullen, 1995) were small and non-significant $\left(M_{\text {change }}=0.48 \pm 5.80\right.$, $p=0.683$ ). Changes in health related physical fitness during the intervention all reflected improvements in physical fitness, but only the isometric push-up showed a significant effect $\left(M_{\text {change }}=4.57 \pm 9.20, p=0.020\right)$. There was also a statistically significant reduction in BMI z-score ( $\left.M_{\text {change }}=-0.20 \pm 0.44, p=0.027\right)$, but changes were due to a significant increase in participant height during the 12 weeks $\left(M_{\text {change }}=2.53 \pm 1.22 \mathrm{~cm}, p<0.001\right)$. No significant changes were observed for any physical activity variable $(p>0.05$; see Table 4).

Competency in fundamental motor skills was associated with multiple aspects of health-related physical fitness and physical activity. At baseline, TGMD-3 Gross Motor Index was positively correlated with performance on modified curl-ups $(r=0.57, p<0.01)$, isometric push-up $(r=0.42$, $p<0.05)$, standing long jump $(r=0.71, p<0.001)$, and $20 \mathrm{~m}$ PACER test $(r=0.52, p<0.01)$. All other associations between FMS competency and body composition or physical activity were small and non-significant $(p>0.05)$. Across the intervention, two notable associations were identified. Improvement in locomotor skills were negatively correlation with change in WHR $(r=-0.43, p<0.05)$. Improvement in the Gross Motor Index was also positively associated with improvements in both moderate physical activity $(r=0.60$, $p<0.01)$ and MVPA $(r=0.49, p<0.05)$. The complete correlation matrix is presented in Supplementary Table S2.

To address the secondary study objective, correlations between ADOS severity scores and intervention outcomes were examined (see Table 5). At baseline, social affect and restrictive and repetitive behaviors were both negatively associated with locomotor skills $(p<0.05)$, indicating greater FMS competency among children with lesser ASD symptoms. Waist to hip ratio was positively correlated with the ADOS comparison score $(r=0.89, p<0.01)$
Table 3 Intervention effect on fundamental motor skills and health related physical fitness

\begin{tabular}{lcccccc}
\hline & $n$ & $\begin{array}{l}\text { Pre } \\
M(S D)\end{array}$ & $\begin{array}{l}\text { Post } \\
M(S D)\end{array}$ & $\begin{array}{l}\text { Change } \\
M(S D)\end{array}$ & $p$ & $d_{z}$ \\
\hline FMS & & & & & & \\
$\quad$ Locomotor & 25 & $17.04(13.81)$ & $17.52(14.05)$ & $0.48(5.80)$ & .683 & .083 \\
Ball Skills & 25 & $13.96(11.90)$ & $19.24(12.52)$ & $5.28(5.62)$ & $<.001 *$ & .938 \\
HRPF & & & & & & \\
BMI z-score & 25 & $1.11(1.53)$ & $0.90(1.65)$ & $-0.20(0.44)$ &. $\mathbf{0 2 7} *$ & .474 \\
Waist-hip ratio & 25 & $1.23(1.70)$ & $0.83(0.11)$ & $-0.40(1.69)$ & .245 & .239 \\
Modified curl ups (\#) & 25 & $2.12(3.41)$ & $3.16(5.38)$ & $1.04(3.80)$ & .184 & .273 \\
Isometric push up (s) & 25 & $3.57(9.36)$ & $8.14(17.23)$ & $4.57(9.20)$ &. $\mathbf{0 2 0} *$ & .497 \\
Standing long jump (\#) & 25 & $35.71(31.40)$ & $41.92(40.24)$ & $6.21(31.55)$ & .335 & .197 \\
20 m PACER (laps) & 25 & $2.28(1.74)$ & $2.32(2.10)$ & $0.04(1.93)$ & .918 & .021 \\
\hline
\end{tabular}

Paired sample t-tests with $d_{\mathrm{z}}$ effect size. Descriptive statistics are presented as mean (standard deviation). FMS fundamental motor skills, HRPF health related physical fitness, body mass index

$* \mathrm{p}<.05$, bolded 
Table 4 Intervention effect on physical activity

\begin{tabular}{lllllll}
\hline & $n$ & Pre & $\begin{array}{l}\text { Post } \\
M(S D)\end{array}$ & $\begin{array}{l}\text { Change } \\
M(S D)\end{array}$ & $p$ & $d_{z}$ \\
\hline Sedentary & 18 & $381.46(103.37)$ & $327.51(141.58)$ & $53.96(131.59)$ & .100 & .410 \\
Light & 18 & $145.77(41.61)$ & $129.10(67.68)$ & $16.67(65.34)$ & .294 & .255 \\
Moderate & 18 & $42.36(14.00)$ & $41.54(29.79)$ & $0.82(30.82)$ & .912 & .027 \\
Vigorous & 18 & $23.12(12.17)$ & $21.55(16.57)$ & $1.56(17.97)$ & .717 & .087 \\
MVPA & 18 & $65.48(24.34)$ & $63.10(43.90)$ & $2.38(47.15)$ & .833 & .050 \\
\hline
\end{tabular}

Paired sample t-tests with $d_{\mathrm{z}}$ effect size. Descriptive statistics are presented as mean (standard deviation) of PA data in average minutes per day. MVPA: moderate to vigorous physical activity

$* \mathrm{p}<.05$ (none observed)
Table 5 Correlations between ASD symptoms, FMS, and HRPF at baseline

\begin{tabular}{|c|c|c|c|}
\hline & $\begin{array}{l}\text { Social } \\
\text { affect }\end{array}$ & $\begin{array}{l}\text { Restrictive \& } \\
\text { repetitive behav- } \\
\text { iors }\end{array}$ & $\begin{array}{l}\text { ADOS-2 } \\
\text { comparison } \\
\text { score }\end{array}$ \\
\hline \multicolumn{4}{|l|}{ FMS } \\
\hline Locomotor & $-.485 *$ & $-.600 * *$ & -.316 \\
\hline Ball skills & $-.494 *$ & $-.556 * *$ & -.296 \\
\hline Gross motor index & $-.684 * *$ & $-.665 * *$ & $-.418 *$ \\
\hline \multicolumn{4}{|l|}{ HRPF } \\
\hline BMI z-score & -.161 & .003 & .111 \\
\hline Waist-hip ratio & .388 & .381 & $.882 * *$ \\
\hline Modified curl ups (\#) & -.241 & -.344 & -.113 \\
\hline Isometric push up (s) & -.125 & -.154 & -.044 \\
\hline Standing long jump (\#) & -.363 & $-.430 *$ & -.296 \\
\hline 20 m PACER (laps) & -.187 & -.131 & -.173 \\
\hline
\end{tabular}

$n=25$. Pearson bivariate correlations at baseline. ADOS-2: Autism Diagnostic Observation Schedule, $2^{\text {nd }}$ edition (Lord et al., 2012); $F M S$ fundamental motor skills, $H R P F$ health related physical fitness, $B M I$ body mass index. Locomotor and ball skills are raw scores. Gross Motor Index is a scaled score

$* \mathrm{p}<.05, * * \mathrm{p}<.01, * * * \mathrm{p}<.001$, bolded

and standing long jump performance was negatively correlated with restrictive and repetitive behaviors $(r=-0.43$, $p<0.05)$. No other outcomes of HRPF were correlated with ADOS scores at baseline $(p>0.05)$.

ASD symptoms were also associated with some effects of the intervention (see Table 6). Change scores $(\Delta)$ in BMI z-score were positively associated with both social affect $(r=0.41, p<0.05)$ and restrictive and repetitive behaviors $(r=0.42, p<0.05)$. Change in waist to hip ratio was negatively associated with ADOS comparison score ( $r=-0.87, p<0.01)$ and change in $20 \mathrm{~m}$ PACER performance was negatively associated with both social affect $(r=-0.40, p<0.05)$ and restrictive and repetitive behaviors $(r=-0.55, p<0.01)$. All other associations with changes in FMS and HRPF were not significant $(p>0.05)$.
Table 6 Correlations between ASD symptoms and intervention outcomes

\begin{tabular}{lrlc}
\hline & $\begin{array}{l}\text { Social } \\
\text { affect }\end{array}$ & $\begin{array}{l}\text { Restrictive \& } \\
\text { repetitive behav- } \\
\text { iors }\end{array}$ & $\begin{array}{l}\text { ADOS-2 } \\
\text { comparison } \\
\text { score }\end{array}$ \\
\hline FMS & & & \\
$\Delta$ Locomotor & .048 & .100 & .247 \\
$\Delta$ Ball skills & -.069 & -.185 & -.100 \\
$\Delta$ Gross motor index & .382 & .329 & .218 \\
HRPF & & & .091 \\
$\Delta$ BMI z-score & $\mathbf{. 4 0 6 *}$ & $\mathbf{. 4 1 8 *}$ & $-.869 * *$ \\
$\Delta$ Waist-hip ratio & -.371 & -.368 & -.098 \\
$\Delta$ Modified curl ups (\#) & -.036 & -.310 & .338 \\
$\Delta$ Isometric push up (s) & -.088 & .027 & .133 \\
$\Delta$ Standing long jump & .013 & -.059 & -.169 \\
$\quad$ \#) & & & \\
$\Delta$ 20 m PACER (laps) &.$- \mathbf{4 0 2 *}$ &.$- \mathbf{5 5 3 * *}$ & \\
\hline
\end{tabular}

$n=25$. Pearson bivariate correlations of intervention outcomes (i.e. change scores, $\Delta$ ) from pre to post intervention. ADOS-2 Autism Diagnostic Observation Schedule, 2nd edition (Lord et al. 2012), $F M S$ fundamental motor skills, $H R P F$ health related physical fitness, $B M I$ body mass index. Locomotor and ball skills are raw scores. Gross Motor Index is a scaled score

$* \mathrm{p}<.05, * * \mathrm{p}<.01, * * * \mathrm{p}<.001$, bolded

Correlations between ADOS scores and physical activity within the smaller sample with physical activity $(n=18)$ were also not statistically significant $(p>0.05)$ at either baseline or across the intervention (see Supplementary Table S3).

\section{Discussion}

Given the pervasive nature of social communication deficits, an often-overlooked component of early intervention includes the disparities in health that disproportionately impact this population. This study followed recommendations for early intervention consistent with an empirically supported behavioral framework (CPRT; Suhrheinrich et al. 
2011), and several outcomes from the current study are supported in previous research (Ketcheson et al. 2017). However, this study addresses several limitations in motor behavior interventions for children on the autism spectrum. First, study participants were between 3 and 5 years of age which represents a younger group of children across a narrower age range compared to other intervention research (Najafabadi et al. 2018; Pan 2010; Toscano et al. 2018), moreover, this study examined multiple components of health including FMS, PA, HRPF and weight status. With respect to the primary objective, to determine the impact of a PA intervention on multiple components of health; all motor and fitness measures showed progress from pre to post intervention, with significant improvements found in ball skills and the isometric push up. The methodology and measures included in this study represent an important next step in the design and delivery of motor behavior interventions for young children on the autism spectrum.

Results from our primary objective are interesting to consider within the developmental framework of Dynamic Systems Theory (DST; Thelen and Smith 1998). DST purports that patterns of motor behavior can be stable or unstable. Stable patterns of behavior are skills which have been acquired and reinforced overtime, and as a result, the pattern of behavior is stable and difficult to change. Unstable patterns of behavior are skills which are easily perturbed given children may not have been afforded an opportunity for acquisition or reinforcement. It is plausible that ball skills represent unstable patterns of behavior, especially when considering environmental supports that facilitate this type of skill acquisition and the potential lack of opportunity for 'at home' instruction requiring sports equipment. While authors note these conclusions may be speculative, it is important to consider the urban environment where the intervention was operationalized. Current research would support that additional barriers exist for opportunities to participate in PA experiences within such environments. Further complicating matters are the unique learning considerations required when instructing children on the autism spectrum.

Another consideration regarding findings from our primary objective is the process through which children acquire FMS. There is empirical support which suggests FMS no not develop naturally, instead the acquisition of FMS requires instruction and practice (Logan et al. 2012). Given the period of developmental plasticity that exists within the preschool years (National Scientific Council on the Developing Child 2007), embedding motor behavior within early intervention can provide young children with multiple opportunities to acquire and refine FMS. These opportunities overtime will build upon one another forming a foundation to maximize their learning of additional motor skills. Within the context of a PA intervention, these early opportunities for skill acquisition form experience-dependent neural connections that can be strengthened with instruction and practice. As a young child gains competence in their performance of FMS, the patterns of behavior become more stable, and connections are strengthened. Whereas movement patterns that are used less frequently are not yet stable, and without intervention are eliminated through a process of developmental pruning. Given children on the autism spectrum are afforded fewer opportunities for the acquisition of FMS, the design and delivery of motor behavior interventions early on in development should be an important consideration within a DST developmental framework.

Next, there is empirical support to suggest that in neurotypical children motor proficiency in balls skills during childhood are a known contributor to levels of PA and HRPF during adolescence. Specifically, proficiency performing ball skills between 7 and 11 years of age was associated with MVPA during adolescence, with the strongest predictor being later participation in vigorous intensity PA (Barnett et al. 2009). Proficiency performing ball skills between the ages of 4-6 years and 7-11 years is also a significant predictor of fitness during adolescence (Barnett et al. 2008; Vlahov et al. 2014). Next, while only ball skills improved post intervention, authors anticipate with a longitudinal design an increase in locomotor skills would have been observed. This is important to consider given that greater proficiency performing locomotor skills is associated with higher levels of vigorous intensity PA among 3-4 year old children (Webster et al. 2019). While the majority of empirical support for relationships between motor skill proficiency and participation in health enhancing PA has included neurotypical children, future studies should consider replication of longitudinal methodology to determine if outcomes are similar for children on the autism spectrum. Moreover, results from the current study should form the catalyst for additional longitudinal research examining the strengthening of relationships between components of health over developmental time.

When considering the outcomes related to PA within this study, it is important to recognize that levels of PA are established early on in development and therefore may have been established as preferred patterns of behavior which are difficult to change in a relatively short amount of time (Wilkin 2011). In fact, current study findings are supported by Ketcheson et al. (2017) that examined changes to objectively measured PA following an intensive short-term motor behavior intervention. Moreover, cross-sectional research suggests that as children on the autism spectrum transition to adolescence, their participation in health enhancing PA declines (Jones et al. 2017; MacDonald et al. 2011), which highlights the importance of establishing positive PA behaviors during childhood. Similar to the acquisition of FMS, it is important to consider the cascading impacts of health enhancing PA throughout development. Given PA typically includes whole body movements, where opportunities to 
form and strengthen connections are available early on in development that may support simultaneous improvements to health.

As noted in the introduction, very few studies to date have examined the HRPF of children on the autism spectrum (Srinivasan et al. 2014; Collins and Staples 2017; Staples et al. 2020). Previous research has shown that children on the autism spectrum between the ages of 7-12 years demonstrate significantly improved levels of HRPF following 10 weeks of participation in PA programming (Collins and Staples 2017). The current study reports significant improvements in only the isometric push up, which is not surprising as the final few minutes of each session were dedicated to the practice of yoga poses. The poses required the combination of different muscle groups where children would have been afforded the opportunity to practice their balance and acquire upper body strength to sustain the isometric push up. These results demonstrate that with targeted practice, significant improvements are possible, but suggest that it may take more time for younger children who are just developing physical fitness capacities to make these gains. In order to influence multiple measures of HRPF, long-term interventions are needed to influence the maintenance and improvement in all health outcomes. These results suggest patterns of behavior can be positively impacted, further solidifying the importance of establishing behaviors that support health enhancing PA behavior early in development. Moreover, given the potentially reciprocal nature of physical activity and physical fitness (Robinson et al. 2015; Stodden et al. 2014) designing early interventions that promote participation in PA may simultaneously improve trajectories of health. Future service providers and early interventionists should provide structured learning experiences where opportunities to reinforce participation in PA will establish improved trajectories of health over developmental time.

With respect to our secondary objective, to examine social determinants of motor behavior (including motor skills) and ASD symptoms at baseline, significant associations were found between social communication, restricted repetitive behaviors and motor skills. Given a child's level of social attention impacts the amount of motor skill acquisition, it is not surprising that children with higher social attention at the beginning of the intervention made the most significant gains in motor outcomes. Authors note that the opportunity to engage in positive and shared emotional experiences during the PA intervention, may have provided a pathway where the experience dependent opportunities facilitate greater motor gains among children who have greater social attention. Next, no associations were found in HRPF (with the exception of waist to hip ratio) and pre to post PA. Given previous research examining changes to PA over short-term interventions, these results are not surprising (Ketcheson et al. 2017). Changes to PA are complex, oftentimes requiring comprehensive lifestyle changes, proving difficult to alter over the short term. In sum, current study findings suggest a careful understanding of core deficits at baseline may lend support for a more individualized approach during motor intervention instruction. In this way, early interventionists can carefully plan for multitiered instruction whereby the facilitation of small or large group work is appropriate to a child's developmental level, and task requirements are incrementally increased throughout the throughout the intervention.

Intervention best practices addressing core deficits have been widely acknowledged and disseminated (Zwaigenbaum et al. 2015), still lacking are guidelines for early interventions that address health outcomes. The majority of physical activity or exercise studies that include individuals on the autism spectrum have examined outcomes as it relates to ASD symptoms or specific behaviors (Bremer et al. 2016; Ruggeri et al. 2020). In fact, there are no known published studies that have reported results on multiple components of health following PA interventions. The short-term outcomes of this study are promising and should lend support for the inclusion of motor behavior within the early intervention prescription for young children on the autism spectrum. Further, carefully designed longitudinal studies would provide insight into changing trajectories in health that occur over developmental time as a result of a PA intervention.

\section{Limitations}

The original design of the study included a longitudinal intervention with multiple time points of data collection, in this way, we hypothesized improvements would be observed in all components of health by the conclusion of the 2-year intervention. Even despite the truncated study, we were encouraged with the results that were found with a relatively small dosage. We anticipate with a longitudinal design improvement across all components of health would be observed. Next, this study did not include a control group and we acknowledge this as a study limitation given generalizations may be difficult to discern. However, given the limited research examining the effectiveness of motor behavior interventions within a narrow age range, that address components of health, this study offers future researchers an opportunity for replication and longitudinal study design. Finally, the current study examined standardized domain scores from the ADOS including SA and RRB to examine if social communication scores at baseline were related improvements in motor behavior. The authors acknowledge more appropriate measures of social communication exist, for example, the Social Responsive Scale (Constantino and Gruber 2012), and the Vineland Adaptive Behavioral Scales-3 (Sparrow et al. 2016). However, 
it should be noted that a significant finding from this study were the relationships observed between fundamental motor skills and measures of SA and RRB. Therefore, current study findings should stimulate continued examination between social determinants of motor behavior and social communication employing comprehensives measures of socialization.

\section{Conclusions}

The delays that children on the autism spectrum experience span across several developmental domains including impairments in motor behavior. The results of this study demonstrate that among young children on the autism spectrum, components of health can be improved following a community-based PA early intervention. In order to address the health disparities in children on the autism spectrum, future researchers are encouraged to design longitudinal motor behavior interventions that begin early on as a way to promote positive trajectories of health throughout development.

Acknowledgments This research was generously supported by two grants, the W.K. Kellogg Foundation [P0131868] as well as the Ralph Wilson Legacy Foundation [2019-0323].

Author Contributions All authors whose names appear on the submission made substantial contributions to the conception or design of the work; or the acquisition, analysis, or interpretation of data; drafted the work or revised it critically for important intellectual content; approved the version to be published; and agree to be accountable for all aspects of the work in ensuring that questions related to the accuracy or integrity of any part of the work are appropriately investigated and resolved.

\section{Compliance with Ethical Standards}

Conflict of interest The authors declare that they have no conflict of interest.

\section{References}

America, S. H. A. P. E., Couturier, L., Chepko, S., \& Holt, S. A. (2014). National standards \& grade-level outcomes for K-12 physical education. Champaign: Human Kinetics.

Barnett, L. M., van Beurden, E., Morgan, P. J., Brooks, L. O., \& Beard, J. R. (2009). Childhood motor skill proficiency as a predictor of adolescent physical activity. The Journal of adolescent health : official publication of the Society for Adolescent Medicine, 44(3), 252-259. https://doi.org/10.1016/j.jadohealth.2008.07.004.

Barnett, L. M., van Beurden, E., Morgan, P. J., Brooks, L. O., \& Beard, J. R. (2008). Does childhood motor skill proficiency predict adolescent fitness? Medicine and Science in Sports and Exercise, 40, 2137-2144. https://doi.org/10.1249/MSS.0b013e31818160d3.

Bhat, A. N., Landa, R. J., \& Galloway, J. C. (2011). Current perspectives on motor functioning in infants, children, and adults with autism spectrum disorders. Physical therapy, 91(7), 1116-1129. https://doi.org/10.2522/ptj.20100294.
Bremer, E., Balogh, R., \& Lloyd, M. (2015). Effectiveness of a fundamental motor skill intervention for 4-year-old children with autism spectrum disorder: A pilot study. Autism, 19(8), 980-991.

Bremer, E., Crozier, M., \& Lloyd, M. (2016). A systematic review of the behavioural outcomes following exercise interventions for children and youth with autism spectrum disorder. Autism, 20(8), 899-915.

Broder-Fingert, S., Brazauskas, K., Lindgren, K., Iannuzzi, D., \& Van Cleave, J. (2014). Prevalence of overweight and obesity in a large clinical sample of children with autism. Academic pediatrics, 14(4), 408-414.

Butte, N. F., Wong, W. W., Lee, J. S., Adolph, A. L., Puyau, M. R., \& Zakeri, I. F. (2014). Prediction of energy expenditure and physical activity in preschoolers. Medicine and science in sports and exercise, 46(6), 1216-1226. https://doi.org/10.1249/ MSS.0000000000000209.

Collins, K., \& Staples, K. (2017). The role of physical activity in improving physical fitness in children with intellectual and developmental disabilities. Research in developmental disabilities, 69, 49-60.

Centers for Disease Control and Prevention (CDC). Clinical growth charts. www.cdc.gov/growthcharts/clinical_charts.htm.

Constantino, J. N., Gruber, C. P. (2012). The Social Responsiveness Scale Manual, Second Edition (SRS-2). Los Angeles, CA: Western Psychological Services.

Curtin, C., Bandini, L. G., Perrin, E. C., et al. (2005). Prevalence of overweight in children and adolescents with attention deficit hyperactivity disorder and autism spectrum disorders: a chart review. BMC Pediatrics. https://doi.org/10.1186/1471-2431-5-48.

Flanagan, J. E., Landa, R., Bhat, A., \& Bauman, M. (2012). Head lag in infants at risk for autism: a preliminary study. The American journal of occupational therapy: official publication of the American Occupational Therapy Association, 66(5), 577-585. https:// doi.org/10.5014/ajot.2012.004192.

Gehricke, J. G., Chan, J., Farmer, J. G., Fenning, R. M., SteinbergEpstein, R., Misra, M., \& Neumeyer, A. M. (2020). Physical activity rates in children and adolescents with autism spectrum disorder compared to the general population. Research in Autism Spectrum Disorders, 70, 101490.

Gillette, M. L., Borner, K. B., Nadler, C. B., Poppert, K. M., Odar Stough, C., Swinburne Romine, R., \& Davis, A. M. (2015). Prevalence and Health Correlates of Overweight and Obesity in Children with Autism Spectrum Disorder. Journal of developmental and behavioral pediatrics: JDBP, 36(7), 489-496. https://doi. org/10.1097/DBP.0000000000000198.

Gladfelter, A., Johnson, E., \& Odeh, C. (2020). Parent perceptions of social behaviors associated with autism spectrum disorder are related to motor skills. Communication Disorders Quarterly, 41(3), 193-196.

Gotham, K., Pickles, A., \& Lord, C. (2009). Standardizing ADOS scores for a measure of severity in autism spectrum disorders. Journal of autism and developmental disorders, 39(5), 693-705.

Healy, S., Nacario, A., Braithwaite, R. E., \& Hopper, C. (2018). The effect of physical activity interventions on youth with autism spectrum disorder: A meta-analysis. Autism research : official journal of the International Society for Autism Research, 11(6), 818-833. https://doi.org/10.1002/aur.1955.

Healy, S., Aigner, C. J., \& Haegele, J. A. (2019). Prevalence of overweight and obesity among US youth with autism spectrum disorder. Autism, 23(4), 1046-1050.

Hill, A. P., Zuckerman, K. E., \& Fombonne, E. (2015). Obesity and Autism. Pediatrics, 136(6), 1051-1061. https://doi.org/10.1542/ peds.2015-1437.

Jasmin, E., Couture, M., McKinley, P., Reid, G., Fombonne, E., \& Gisel, E. (2009). Sensori-motor and daily living skills of preschool 
children with autism spectrum disorders. Journal of autism and developmental disorders, 39(2), 231-241.

Jones, R. A., Downing, K., Rinehart, N. J., Barnett, L. M., May, T., McGillivray, J. A., et al. (2017). Physical activity, sedentary behavior and their correlates in children with autism spectrum disorder: A systematic review. PLoS ONE. https://doi.org/10.1371/ journal.pone. 0172482 .

Ketcheson, L., Hauck, J., \& Ulrich, D. (2017). The effects of an early motor skill intervention on motor skills, levels of physical activity, and socialization in young children with autism spectrum disorder: A pilot study. Autism, 21(4), 481-492.

Ketcheson, L., Hauck, J. L., \& Ulrich, D. (2018). The levels of physical activity and motor skills in young children with and without autism spectrum disorder, aged 2-5 years. Autism, 22(4), 414423. https://doi.org/10.1177/1362361316683889.

King-Dowling, S., Proudfoot, N. A., Cairney, J., \& Timmons, B. W. (2020). Motor Competence, Physical Activity, and Fitness across Early Childhood. Medicine and Science in Sports and Exercise. https://doi.org/10.1249/mss.0000000000002388.

Kuczmarski, R. J., Ogden, C. L., Grummer-Strawn, L. M., Flegal, K. M., Guo, S. S., Wei, R., et al. (2000). CDC growth charts: United States. Advance Data, 314, 1-27.

Levy, S. E., Pinto-Martin, J. A., Bradley, C. B., Chittams, J., Johnson, S. L., Pandey, J., et al. (2019). Relationship of Weight Outcomes, Co-Occurring Conditions, and Severity of Autism Spectrum Disorder in the Study to Explore Early Development. The Journal of pediatrics, 205, 202-209. https://doi.org/10.1016/j.jpeds 2018.09.003.

Lloyd, M., MacDonald, M., \& Lord, C. (2013). Motor skills of toddlers with autism spectrum disorders. Autism, 17(2), 133-146.

Logan, S. L., Nicholas, J. S., Carpenter, L. A., King, L. B., GarrettMayer, E., \& Charles, J. M. (2012). High prescription drug use and associated costs among Medicaid-eligible children with autism spectrum disorders identified by a population-based surveillance network. Annals of epidemiology, 22(1), 1-8. https://doi. org/10.1016/j.annepidem.2011.10.007.

Logan, S. W., Webster, E. K., Getchell, N., Pfeiffer, K. A., \& Robinson, L. E. (2015). Relationship between fundamental motor skill competence and physical activity during childhood and adolescence: A systematic review. Kinesiology Review, 4, 416-426. https://doi. org/10.1123/kr.2013-0012.

Lord, C., Rutter, M., DiLavore, P. C., Risi, S., Gotham, K., \& Bishop, S. (2012). Autism Diagnostic Observation Schedule (2nd ed.). Torrence, CA: Western Psychological Services.

MacDonald, M., Esposito, P., \& Ulrich, D. (2011). The physical activity patterns of children with autism. BMC Research Notes, 4, 422. https://doi.org/10.1186/1756-0500-4-422.

MacDonald, M., Lord, C., \& Ulrich, D. (2013). The relationship of motor skills and adaptive behavior skills in young children with autism spectrum disorders. Research in autism spectrum disorders, 7(11), 1383-1390. https://doi.org/10.1016/j. rasd.2013.07.020

Maenner, M. J., Shaw, K. A., Baio, J., Washington, A., Patrick, M., DiRienzo, M., et al. (2020). Prevalence of Autism Spectrum Disorder Among Children Aged 8 Years-Autism and Developmental Disabilities Monitoring Network, 11 Sites, United States, 2016. Morbidity and mortality weekly report Surveillance summaries, 69(4), 1-12. https://doi.org/10.15585/mmwr.ss6904a1.

Matthews, C. E., Hagströmer, M., Pober, D. M., \& Bowles, H. R. (2012). Best practices for using physical activity monitors in population-based research. Medicine and Science in Sports and Exercise, 44, S68-S76. https://doi.org/10.1249/MSS.0b013e3182 $399 \mathrm{e} 5 \mathrm{~b}$.

Michigan's Exemplary Physical Education Curriculum. (2000). EPEC lessons-Grades K, 1, 2, 3, 4, 5, and user's manual.
Mullen, E. M. (1995). Mullen scales of early learning (pp. 58-64). Circle Pines, MN: AGS.

Nahmias, A. S., Pellecchia, M., Stahmer, A. C., \& Mandell, D. S. (2019). Effectiveness of community-based early intervention for children with autism spectrum disorder: a meta-analysis. Journal of child psychology and psychiatry, and allied disciplines, 60(11), 1200-1209. https://doi.org/10.1111/jcpp.13073.

Najafabadi, M. G., Sheikh, M., Hemayattalab, R., Memari, A. H., Aderyani, M. R., \& Hafizi, S. (2018). The effect of SPARK on social and motor skills of children with autism. Pediatrics \& Neonatology, 59(5), 481-487.

National Autism Center. (2009a). Evidence-based practice and autism in the schools: A guide to providing appropriate interventions to students with autism spectrum disorders. Randolph, MA: Author.

National Autism Center. (2009b). National standards report. Randolph, MA: Author.

National Scientific Council on the Developing Child. (2007). The science of early childhood development: Closing the gap between what we know and what we do. http://www.developingchild.net

Ogden, C. L., Carroll, M. D., Lawman, H. G., Fryar, C. D., KruszonMoran, D., Kit, B. K., \& Flegal, K. M. (2016). Trends in obesity prevalence among children and adolescents in the United States, 1988-1994 through 2013-2014. Journal of the American Medical Association, 315, 2292-2299. https://doi.org/10.1001/ jama.2016.6361.

Pan, C. Y. (2010). Effects of water exercise swimming program on aquatic skills and social behaviors in children with autism spectrum disorders. Autism, 14(1), 9-28.

Pfeiffer, K. A., Dowda, M., McIver, K. L., \& Pate, R. R. (2009). Factors related to objectively measured physical activity in preschool children. Pediatric Exercise Science, 21(2), 196-208. https://doi. org/10.1123/pes.21.2.196.

Pitchford, E. A., \& Webster, E. K. (2020). Clinical Validity of the Test of Gross Motor Development-3 in Children With Disabilities from the US National Normative Sample. Adapted Physical Activity Quarterly. https://doi.org/10.1123/apaq.2020-0023.

Provost, B., Lopez, B. R., \& Heimerl, S. (2007). A comparison of motor delays in young children: autism spectrum disorder, developmental delay, and developmental concerns. Journal of autism and developmental disorders, 37(2), 321-328.

Pusponegoro, H. D., Efar, P., Soebadi, A., Firmansyah, A., Chen, H. J., \& Hung, K. L. (2016). Gross motor profile and its association with socialization skills in children with autism spectrum disorders. Pediatrics \& Neonatology, 57(6), 501-507.

Rich, C., Geraci, M., Griffiths, L., Sera, F., Dezateux, C., \& CortinaBorja, M. (2013). Quality control methods in accelerometer data processing: Definining minimum wear time. PLOS ONE, 8(6), e67206. https://doi.org/10.1371/journal.pone.0067206.

Robinson, L. E., Stodden, D. F., Barnett, L. M., Lopes, V. P., Logan, S. W., Rodrigues, L. P., \& D'Hondt, E. (2015). Motor competence and its effect on positive developmental trajectories of health. Sports Medicine, 45, 1273-1284. https://doi.org/10.1007/s4027 9-015-0351-6.

Ruggeri, A., Dancel, A., Johnson, R., \& Sargent, B. (2020). The effect of motor and physical activity intervention on motor outcomes of children with autism spectrum disorder: A systematic review. Autism, 24(3), 544-568. https://doi.org/10.1177/1362361319 885215.

Sparrow, S. S., Cichetti, D. V., Saulnier, C. A. (2016). Vineland Adaptive Behavior Scales, Third edition (Vineland-3) manual. Bloomington, MN: PsychCorp.

Stodden, D. F., Goodway, J. D., Langendorfer, S. J., Roberton, M. A., Rudisill, M. E., Garcia, C., \& García, L. F. (2008). A Developmental Perspective on the Role of Motor Skill Competence in Physical Activity: An Emergent Relationship. Quest, 60, 290-306. 
Stodden, D. F., Gao, Z., Goodway, J. D., \& Langendorfer, S. J. (2014). Dynamic relationships between motor skill competence and health-related fitness in youth. Pediatric Exercise Science, 26, 231-241. https://doi.org/10.1123/pes.2013-0027.

Suhrheinrich, J., Reed, S., Schreibman, L., \& Bolduc, C. (2011). Classroom pivotal response teaching for children with autism. New York: Guilford Press.

Staples, K. L., MacDonald, M., \& Zimmer, C. (2012). Assessment of motor behavior among children and adolescents with autism spectrum disorder. International Review of Research in Developmental Disabilities, 42, 179-214. https://doi.org/10.1016/B9780-12-394284-5.00007-3.

Staples, K. L., \& Reid, G. (2010). Fundamental movement skills and autism spectrum disorders. Journal of autism and developmental disorders, 40(2), 209-217.

Staples, K. L., Ketcheson, L., \& Parks, A. C. (2020). Understanding health disparities among 3 to 9 year old children with autism spectrum disorder. Sport and Exercise Psychology. https://doi. org/10.1123/jsep.2020-0172.

Staples, K. L., Pitchford, E. A., \& Ulrich, D. A. (2020). The instructional sensitivity of the Test of Gross Motor Development - 3 to detect changes in performance for young children with and without Down syndrome. Adapted Physical Activity Quarterly. https ://doi.org/10.1123/apaq.2020-0047.

Srinivasan, S. M., Pescatello, L. S., \& Bhat, A. N. (2014). Current perspectives on physical activity and exercise recommendations for children and adolescents with autism spectrum disorders. Physical therapy, 94(6), 875-889.

Teitelbaum, P., Teitelbaum, O., Nye, J., Fryman, J., \& Maurer, R. G. (1998). Movement analysis in infancy may be useful for early diagnosis of autism. Proceedings of the National Academy of Sciences of the United States of America, 95(23), 13982-13987. https ://doi.org/10.1073/pnas.95.23.13982.

Thelen, E., \& Smith, L. B. (1998). Dynamic systems theories. In R. M. Lerner (Ed.), Handbook of child psychology: Theoretical models of human development (5th ed., Vol. 1, pp. 563-634). New York, NY: John Wiley \& Sons.

Thomas, S., Hinkley, T., Barnett, L. M., \& Rinehart, N. (2019). Young children with ASD participate in the same level of physical activity as children without ASD: Implications for early intervention to maintain good health. Journal of Autism and Developmental
Disorders, 49, 3278-3289. https://doi.org/10.1007/s10803-01904026-9.

Toscano, C. V., Carvalho, H. M., \& Ferreira, J. P. (2018). Exercise effects for children with autism spectrum disorder: metabolic health, autistic traits, and quality of life. Perceptual and motor skills, 125(1), 126-146.

Ulrich, D. A. (2018). Test of Gross Motor Development (3rd ed.). Austin, TX: Pro-Ed.

United States Department of Health and Human Services. (2018). Physical Activity Guidelines for Americans (2nd ed.). Washington, DC: U.S. Department of Health and Human Services.

Vlahov, E., Baghurst, T. M., \& Mwavita, M. (2014). Preschool motor development predicting high school health-related physical fitness: a prospective study. Perceptual and motor skills, 119(1), 279-291.

Webster, E. K., Martin, C. K., \& Staiano, A. E. (2019). Fundamental motor skills, screen time, and physical activity in preschoolers. Journal of Sport and Health Science, 8(2), 114-121. https://doi. org/10.1016/j.jshs.2018.11.006.

Wilkin, T. J. (2011). Can we modulate physical activity in children? International journal of obesity, 35(10), 1270-1276.

Winnick, J. P., \& Short, F. X. (2014). Brockport physical fitness test manual: A health-related assessment for youngsters with disabilities. Champaign, IL: Human Kinetics.

Zuckerman, K. E., Hill, A. P., Guion, K., Voltolina, L., \& Fombonne, E. (2014). Overweight and obesity: prevalence and correlates in a large clinical sample of children with autism spectrum disorder. Journal of autism and developmental disorders, 44(7), 17081719. https://doi.org/10.1007/s10803-014-2050-9.

Zwaigenbaum, L., Bauman, M. L., Choueiri, R., Kasari, C., Carter, A., Granpeesheh, D., \& Pierce, K. (2015). Early intervention for children with autism spectrum disorder under 3 years of age: recommendations for practice and research. Pediatrics, 136(Supplement 1), S60-S81.

Publisher's Note Springer Nature remains neutral with regard to jurisdictional claims in published maps and institutional affiliations. 